\title{
A Study on Maternal Viral Load, CD4 Cell Counts and Time of Mother-to-Child Transmission of HIV-1 in Two Hospitals in Central Ethiopia
}

\author{
Merga Gonfa $^{1}$, Solomon Gebre-Selassie ${ }^{2 *}$ \\ ${ }^{1}$ Adama Science and Technology University, Asella College of Health Sciences, Adama, Ethiopia \\ ${ }^{2}$ Department of Microbiology, Immunology and Parasitology, School of Medicine, Addis Ababa University, \\ Addis Ababa, Ethiopia \\ Email: sesimego@gmail.com, ${ }^{*}$ solomongst@yahoo.com
}

Received 3 May 2014; revised 5 June 2014; accepted 17 June 2014

Copyright (C) 2014 by authors and OALib.

This work is licensed under the Creative Commons Attribution International License (CC BY). http://creativecommons.org/licenses/by/4.0/

(c) (i) Open Access

\section{Abstract}

Background: Vertical transmission of HIV among women who have no access to ART is estimated to be $25 \%-35 \%$ in Africa. Objective: The aim of the study was to assess the time of MTCT of HIV-1 the efficacy of ART on prevention of mother-to-child transmission (MTCT) of HIV-1 in two hospitals. Methods: Prospective cohort study was conducted from November 2011 to May 2012. A total of 162 participants, 81 mothers ( 24 non-breastfeeding and 57 breastfeeding) and 81 infants were involved in the study. Venous blood from mothers and heel blood (dried blood spot) from infants were collected and processed in Adama Regional laboratory. All dried blood spot specimens of infants reactive on ELISA test were assessed by DNA-based polymerase chain reaction tests (DNA PCR) to determine HIV infection. Data were entered and analysed using SPSS version 19 computer software. Results: Five infants were infected with HIV at the end of this study. The overall rate of vertical transmission of HIV was $6.2 \%$. Maternal viral load at delivery was independently associated with both in-utero and intra-partum transmission $(O R=27.0,95 \% \mathrm{CI}, 3.5-210, p=0.001)$. Maternal viral load at 6 weeks of birth and low infant birth weight was associated with intra-partum transmission among breastfeeding mothers $(\mathrm{OR}=25.5,95 \% \mathrm{CI}, 1.14-572, p=0.04)$ and $(\mathrm{OR}=$ 29.6, 95\% CI, 3.2 - 273, $p=0.004$ ), respectively. There were $40 \%$ MTCT of HIV among non ARV drug users and only 3.9\% among those used ARV drugs during their current pregnancy. Conclusion: Strategies planned to reduce maternal viral load during pregnancy can be successful in substantially reducing vertical transmission of HIV. In addition, other contributing factors for MTCT of HIV-1 should be controlled.

\footnotetext{
${ }^{*}$ Corresponding author.
} 


\title{
Keywords
}

\section{Maternal Viral Load, Mother-to-Child Transmission of HIV-1, Perinatal Transmission, ART}

\author{
Subject Areas: HIV, Immunology
}

\section{Introduction}

Mother-to-child transmission (MTCT) of HIV is one of the biggest challenges of the HIV/AIDS pandemic especially in resource constrained settings [1]. MTCT is responsible for about $90 \%$ of HIV infection in children worldwide [2]. In Africa, the burden of the disease accounts for about $90 \%$ of paediatric HIV infections [3]. The risk of mother-to-child transmission of HIV among pregnant women who had no access to antiretroviral treatment is about $25 \%-35 \%$ in Africa [4].

In the absence of prevention, rates of MTCT are estimated to be $25 \%-35 \%$. The use of antiretroviral therapy to reduce mother-to-child transmission of HIV (MTCT) is an important advance in preventing HIV infections in children. When antiretroviral drugs (ARVs) are administered during pregnancy, and breastfeeding is avoided, mother-to-child HIV transmission (MTCT) may occur in fewer than $1 \%$ of pregnancies [5]. In developed countries, the use of ARV as prophylaxis or treatment in addition with elective Caesarean section and avoidance of breastfeeding have reduced the risk of MTCT of HIV from 25\% to 1\% - 5\% [6]. Mullick et al. [2] showed that about $5 \%-10 \%$ of MTCT of HIV occurred as a result of intrauterine transmission, $10 \%-20 \%$ during delivery whereas postnatal MTCT of HIV contributes only $5 \%-20 \%$. WHO recommendation is to reduce the risk of MTCT to less than 5\% in breastfeeding populations from a background risk of $35 \%$, and to less than $2 \%$ in nonbreastfeeding populations from a background risk of $25 \%$, and will ensure increased maternal and child survival [4].

The majority of the burden of HIV infection is concentrated among pregnant women living in low income countries [7]. High maternal viral loads are risk factors for MTCT [2] [8] [9]. Other risk factors that can fuel up MTCT of HIV include breastfeeding, sexually transmitted diseases, vaginal mode of delivery, low CD4 count, advanced maternal HIV disease, young maternal age, and history of stillbirth [10].

Assessing maternal viral load and CD4 cell counts and taking necessary measures relating with time of vertical transmission is an essential to reduce early mortality and morbidity among infants born to HIV-1 positive mothers. Thus, this study tried to determine the proportions and time of MTCT of HIV-1 and evaluate the efficacy of ARV/HAART on prevention of MTCT of HIV-1 in the study areas.

\section{Materials and Methods}

\subsection{Design and Population}

Prospective cohort study was conducted during November 2011 to May 2012 at Adama and Asella hospitals in central Ethiopia. HIV sero-positive pregnant women and their neonates receiving ARV at antenatal care (ANC) services in the two hospitals were eligible for the study. The children received ARV drugs at birth, and were followed up between birth and 10 months. A convenient sampling technique was used to recruit the study unit. A total of eighty one HIV positive mothers with their infants were enrolled in the study.

\subsection{Sample Collection and Processing}

Blood was collected from consented HIV positive pregnant women linked to ART clinics of the hospitals. The blood was collected in two test tubes with EDTA anticoagulant after delivery or during follow-up visit for those HIV positive mothers whose infants birth PCR result was negative. The blood in the first test tube was used to perform CD4 cell count and the second tube was used to measure maternal viral level in the plasma using Abbott Realtime HIV-1 PCR assay. Whole blood was collected from each infants' heel on Whatman 903 DBS collection card within 72 hours of birth or during follow-up time which was used to test in-utero or intrapartum/postpartum mother-to-infant HIV-1 transmission, respectively. Infants' DBS samples were kept at room temperature until HIV-1 DNA PCR tests done. The test utilizes amplification of target DNA by the PCR and nucleic acid hybridi- 
zation for the detection of HIV-1 DNA in human whole blood using AMPLICOR ${ }^{\circledR}$ HIV-1 DNA PCR Test version 1.5 (a qualitative in vitro test for the detection of HIV-1 DNA in human whole blood) Roche Diagnostics System. Specimen were transported to Oromiya Public Health Research laboratory in Adama town for processing within the time limit given in the specimen processing manual or indicated by the test kits manufacturers. Two types of external and one type of internal controls were processed along with infants' DBS samples.

Polymerase chain reaction (PCR) assay (Abbott Real Time HIV-1 RT-PCR) was done for the quantitation of HIV-1 in human plasma from infected individuals for maternal viral load quantfication. Three controls, one HIV-1 negative, one low HIV-1 positive and one high HIV-1 positive were processed along the study participants' samples. These controls came with viral load determination test kits and were included during performing each specimen testing time. Maternal CD4 cells count was performed on the same day of sample collection using FACScalibur flowcytometry (Becton Dickinson FACSCount System). For doubtful result specimens were repeated with new reagent kit following the standard operating procedure (SOP) for the test and manufacturer's protocol.

\subsection{Schedule for Follow-Up and Works Done}

Mother-infant pairs in those whose infants were negative for birth PCR result were under follow-up at six weeks and ten weeks after birth. Each mother-infant pairs gave blood samples at each follow-up time which were to be tested for maternal viral load and CD4 cells level for mothers and the presence of HIV-1 DNA in infant's specimen, respectively. These samples were tested to see mother-to-infant transmission of HIV-1 during intra-partum or postnatally through breastfeeding. Mother whose infant became positive for HIV-1 DNA PCR either at delivery ( $<72$ hrs of delivery, confirms in-utero transmission) or positive DNA PCR result during the first visit which insures intra-partum vertical transmission in non-breastfeeding population or that confirm intra-partum/postpartum transmission in breastfeeding populations would give DBS sample to confirm vertical transmission. The whole process is clearly shown in the workflow chart as follows (Figure 1).

\subsection{Definition of Infant HIV Infection}

Infants were assumed to be infected in uterus if their birth HIV-DNA PCR is tested positive at $<72$ hours of life for both breastfeeding and non-breastfeeding population. They were considered to have acquired HIV during intra-partum if they are diagnosed DNA PCR positive at six weeks of life among non-breastfeeding population. They were considered HIV infected during intra-partum/postnatally if their DNA PCR results were positive at six weeks of life and later on at ten weeks of life in breastfeeding arms.

\subsection{Statistical Analysis}

Data were entered and descriptive analysis was performed using Epi Info version 3.5.1 computer software. Data were exported to SPSS version 19.0 computer software for statistical analysis to elucidate risk factors associated with MTCT of HIV-1. Bivariate and multivariate analysis were made at 95\% confidence interval (IC) to see their association with the hypothesized mother-to-infant transmission rate of HIV-1. Continuous variables are dichotomized within the sample median. Multivariate analyses were performed by using multiple logistic regressions and $95 \% \mathrm{CI}$ were computed to test whether a risk factor is related to time of transmission at $p=0.05$. The attributable risk was determined for maternal viral load and CD4 levels.

\section{Results}

Eighty seven HIV positive mothers delivered at Asella and Adama hospitals during data collection time. Of 87 those mothers who would be expected to participate in the current study, only 81 HIV positive mothers (21 and 60 mother-infant pairs) from Asella and Adama hospitals, respectively, were followed. Because, three motherinfant pairs refused to participate, death of one mother-infant pair occurred after delivery, one mother and one infant died after birth. Of the 87 HIV positive pregnant mothers who delivered at the two hospitals during data collection time, 81 (94.2\%) mothers (29.4\% non-breastfeeding and $70.4 \%$ breastfeeding) had infants with known HIV infection status.

Among 81 mother-infant pairs with known HIV transmission outcome 76 (93.8\%) including 2 (40\%) of the 5 infected infants did have HIV-1DNA PCR test result for specimen collected within 24 hours of delivery. The 


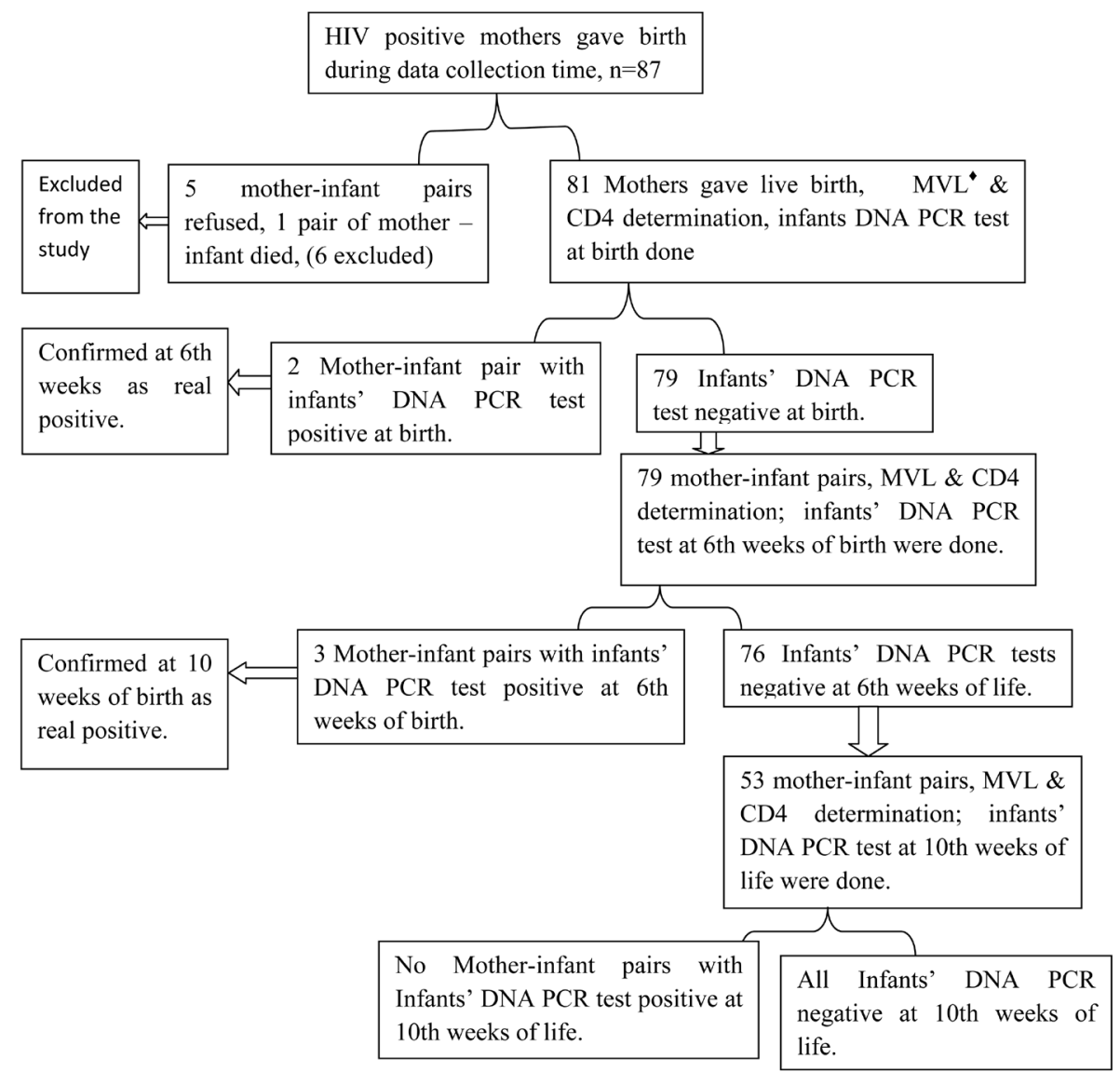

MVL`: Maternal viral load.

Figure 1. Enrollment and follow-up of study participants.

other $4.9 \%$ did have HIV-1 DNA PCR test result for specimens collected at less than 48 hours and the remained 1.2\% had HIV-1 DNA PCR test result for specimen collected at less than 72 hours of birth. From 81 HIV positive pregnant women who participated in this study, 57 (70.4\%) chose exclusive breastfeeding and 24 (29.6\%) mothers did have chose exclusive formula feeding (Figure 2).

The rate of MTCT of HIV varies in relation to different maternal demographic factors such as maternal educational status, marital status, and work category (Table 1).

It was found that $4.9 \%$ MTCT of HIV-1 occurred among married HIV positive women of the current study participant in the general study participant mothers. But, MTCT of HIV was 5.6\% (95\% CI, 1.6 - 13.8) with $2.8 \%$ in-utero and $2.9 \%$ in intra-partum transmission rate among this group. One separated HIV positive mother transmitted HIV to her infant at six weeks of life which accounted for $1.2 \%$ in the total population in this study.

The rate of MTCT of HIV-1 among illiterate HIV positive mothers of the current study participants was $2.5 \%$ in the general population of this study. However, it became 13.3\%, (95\% CI, $1.7-40.5)$, with 6.3\% in-utero and $6.7 \%$ in intra-partum transmission observed when the estimation of the rate of MTCT of HIV is restricted to this group. Another $2.5 \%$ of HIV-1 vertical transmission was determined among the current study participant mothers whose level of education range from grade 5 - 8 in the general population of the current study participant mothers with $0 \%$ in-utero and $8 \%$ in intra-partum transmission rate. One study participant mother whose educational status classified in grade 9 - 10 transmitted HIV to her infant which accounted for $1.2 \%$ MTCT of HIV in the general population and $4.3 \%$ vertical transmission in this group (95\% CI, 0.1 - 21.9). Whereas, MTCT of HIV-1 was not observed among those study participant mothers whose educational status located in the range of grade $1-4$, grade $11-12$ and above grade 12 in this study. Our data could not show clear association between maternal educational status and MTCT of HIV-1; so that, this might be more clarified in other future work.

Total MTCT of HIV (6.2\%) was observed only among house wife study participant HIV positive mothers that 


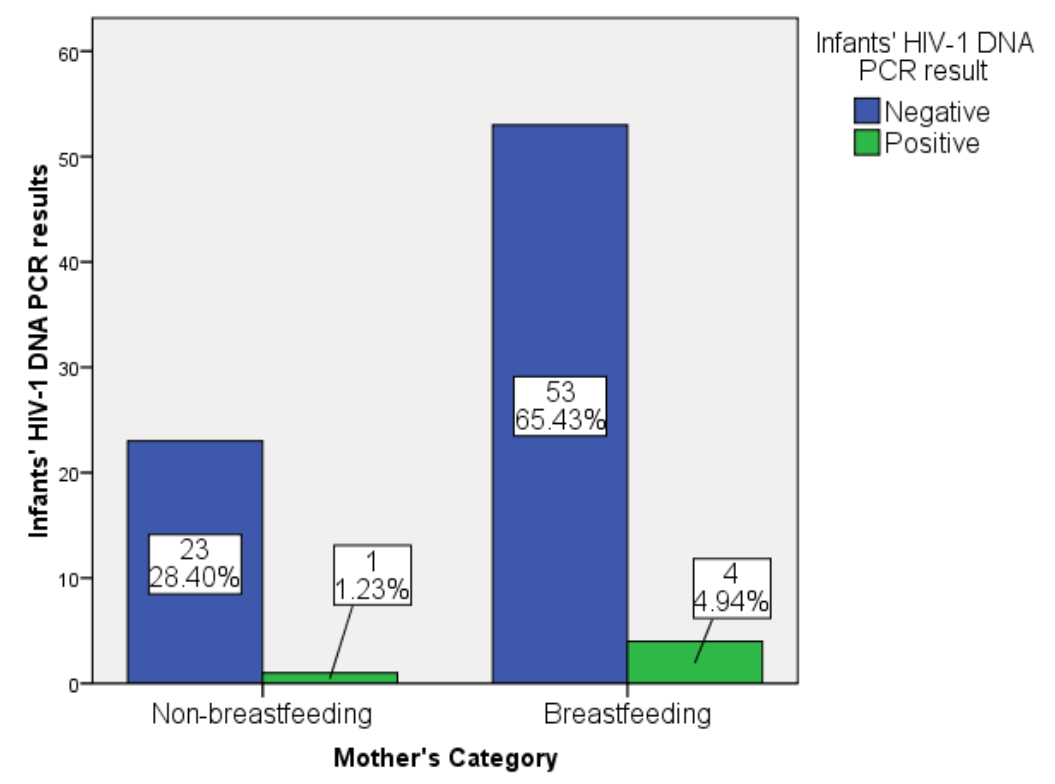

Figure 2. Distribution of MTCT of HIV-1 among breastfeeding and non-breastfeeding study participant mothers at Asella and Adama hospitals, Ethiopia, 2012.

comprise $67.9 \%$ of the overall study participant women. Restricting the determination of rate of MTCT of HIV to this group mothers, we found that the rate of vertical transmission being 9.1\% (95\% CI, 3 - 20), with 3.6\% in-utero and 5.7\% in intra-partum transmission in this study. Of 81 study mothers, 50 (61.7\%) had gave birth before the current pregnancy. Of the 50 mothers, 49 (98\%) had given live birth and 1 (2\%) mother gave still birth. Among these 49 mothers, 33 (67.3\%) mothers had used exclusive breastfeeding (95\% CI, 52.3 - 80.1), 12 (24.5\%) mixed feeding and the others 4 (8.2\%) formula feeding to feed their children before the current pregnancy at the study area. From forty nine live born infants who born before the current pregnancy to these study participant HIV positive mothers, 43 (87.8\%), (95\% CI, 75.2 - 95.4) had known HIV test results at six weeks of life with 23.3\% MTCT of HIV (95\% CI, 11.8 - 38.6). The remained $6(12.2 \%)$ did not have any HIV test result at all in the current study area. As data that were collected from study participant mothers through interviewer administered structured questionnaire indicated (data not shown), this 23.3\% MTCT of HIV was detected among infants born to these HIV positive mothers during the era of single dose nevirapine usage as prophylaxis to prevent MTCT of HIV infection.

Among the current study participant mothers, 76 (93.8\%) mothers had been using antiretroviral drugs 41 (53.9\%) were on ART and 35 (46.1\%) had used zidovudine as prophylaxis to prevent MTCT of HIV during the current pregnancy (95\% CI, 86.2 - 98.0). The remained 5 (6.2\%) of the study participant mothers did not use any ARV-drugs during their current pregnancy (95\% CI, 2 - 13.8) (Table 2).

In the current study $19.8 \%$ of the study participants had been treated for STIs for one to two times and $9.9 \%$ was for malaria for more than two times during their current pregnancy. There was no statistically significant association between maternal STIs or malaria infection and vertical HIV transmission observed in the current study.

For the 81 infants with a known HIV-1 DNA PCR test result at birth, the overall HIV transmission rate was $6.2 \%$. Two infected infants tested HIV-1 positive by DNA PCR assay at birth, giving $2.5 \%$ rate of an in-utero MTCT of HIV infection; (95\% CI, 0.3 - 8.6). Three infected infants were tested HIV-1 negative by HIV-1 DNA PCR at birth and subsequently tested HIV-1 DNA PCR positive at six weeks of life, giving an intra-partum/ postpartum transmission rate of 3.8\% (95\% CI, 0.8 - 10.7) with proportion of $4.2 \%$ among non-breastfeeding and $3.6 \%$ among breastfeeding mothers. When we restricted the determination of in-utero transmission of HIV only to breastfeeding mothers, it was 3.5\% even though, in-utero transmission in the general sample was $2.5 \%$ (Table 3).

HIV-1 DNA PCR testing at birth detected only 2 infants out of 5 infected infants, suggesting that $40 \%$ of HIV transmission took place in in-utero and $60 \%$ transmission was occurred in intra-partum in the current study. 
Table 1. Distribution of total infants' HIV-1 DNA PCR results according to marital, educational and job status of the study participant mothers at Asella and Adama hospitals, Ethiopia, 2012.

\begin{tabular}{|c|c|c|c|}
\hline & Total infants & PCR result & \multirow{2}{*}{ Total, No (\%) } \\
\hline & Negative, No (\%) & Positive, No (\%) & \\
\hline \multicolumn{4}{|c|}{ Marital status of the mothers } \\
\hline Single & $7(8.6)$ & $0(0)$ & $7(8.6)$ \\
\hline Married & $67(82.7)$ & $4(4.9)$ & $71(87.7)$ \\
\hline Separated & $0(0)$ & $1(1.2)$ & $1(1.2)$ \\
\hline Widowed & $1(1.2)$ & $0(0)$ & $1(1.2)$ \\
\hline Divorced & $1(1.2)$ & $0(0)$ & $1(1.2)$ \\
\hline Total & $76(93.8)$ & $5(6.2)$ & $81(100)$ \\
\hline \multicolumn{4}{|c|}{ Educational status of the mothers } \\
\hline Illiterate & $14(17.3)$ & $2(2.5)$ & $16(19.8)$ \\
\hline $1-4$ & $6(7.4)$ & $0(0)$ & $6(7.4)$ \\
\hline $5-8$ & $23(28.4)$ & $2(2.5)$ & 25 (30.9) \\
\hline $9-10$ & $22(27.2)$ & $1(1.2)$ & $23(28.3)$ \\
\hline $11-12$ & $3(3.7)$ & $0(0)$ & $3(3.7)$ \\
\hline$>12$ & $8(9.9)$ & $0(0)$ & $8(9.9)$ \\
\hline Total & $76(93.8)$ & $5(6.2)$ & $81(100)$ \\
\hline \multicolumn{4}{|c|}{ Job category of study participant mothers } \\
\hline House wife & $50(61.7)$ & $5(6.2)$ & $55(67.9)$ \\
\hline Day laborer & $5(6.2)$ & $0(0)$ & $5(6.2)$ \\
\hline Self employer & $12(14.8)$ & $0(0)$ & $12(14.8)$ \\
\hline Government employer & $7(8.6)$ & $0(0)$ & $7(8.6)$ \\
\hline Farmer & $2(2.5)$ & $0(0)$ & $2(2.5)$ \\
\hline Total & 76 (93.8) & $5(6.2)$ & $81(100)$ \\
\hline
\end{tabular}

Table 2. Distribution of total infants' HIV-1 DNA PCR results according to ARV drugs treatment among the study participant mothers at Asella and Adama hospitals, Ethiopia, 2012.

\begin{tabular}{cccc}
\hline & Total infants & HIV-1 DNA & PCR results \\
ARV drugs treatment & Negative, No (\%) & Positive, No (\%) & Total, No (\%) \\
\hline Not used ARV drugs & $3(3.7)$ & $2(2.5)$ & $5(6.2)$ \\
On Prophylaxis/Zidovudin & $34(42)$ & $1(1.2)$ & $35(43.2)$ \\
On ART & $39(48.1)$ & $2(2.5)$ & $41(50.6)$ \\
Total & $76(93.8)$ & $5(6.2)$ & $81(100)$ \\
\hline
\end{tabular}


Table 3. Mother-to-child transmission of HIV-1 according to infant feeding categories of the study participant mothers at Asella and Adama hospitals, Ethiopia, 2012.

\begin{tabular}{|c|c|c|c|c|}
\hline & Non-breastfeeding group & Breastfeeding group & Total No (\%) & OR (95\% CI) \\
\hline Time HIV-1 PCR test done & HIV + No (\%) tested & No HIV+ No (\%) tested & & \\
\hline$<72$ hours (In-utero) & $0 / 24(0)$ & 2/57 (3.5) & $2(2.5)$ & $12.1(1.54-93.5)$ \\
\hline At six weeks (Intra-partum) & $1 / 24(4.2)$ & 2/55 (3.6) & $3(3.7)$ & $25.5(1.14-572)$ \\
\hline At ten weeks ${ }^{*}$ & - & $0 / 55(0)$ & 0 & \\
\hline Total & 1 & 4 & $5(6.2)$ & \\
\hline
\end{tabular}

*(No infection observed).

There was no in-utero and 4.2\% intra-partum MTCT of HIV among non-breastfeeding mothers in the current study. Associating intra-partum transmission with maternal viral load level, $1.9 \%$ of intra-partum MTCT of HIV was occurred among breastfeeding women who had maternal viral load below the sample median $(<9331$ copies $/ \mathrm{ml}$ ) at six weeks of birth (95\% CI, 0.0 - 10.3). The other 33.3\% of intra-partum/post-partum MTCT of HIV took place among breastfeeding mothers who had maternal viral load above the sample median ( $\geq 9331$ copies/ml) at 6 weeks of delivery (95\% CI, 0.8 - 90.6). Infants born to breastfeeding HIV positive mothers had 1.7 times more likely to have positive HIV-1 DNA PCR test result at six weeks of life compared to their counterparts born to non-breastfeeding mothers (Table 3).

High maternal viral load at delivery was independently associated with in-utero transmission so that mothers who had viral load above the sample median ( $\geq 24,665$ copies $/ \mathrm{ml})$ at birth had 12.1 times more likely to have infants infected in-utero compared to their counterparts (OR, 12.1 (95\% CI, 1.54 - 93.5, $p=0.02)$ ). Both high level of maternal viral load greater/equal to the sample median $(\geq 9331$ copies $/ \mathrm{ml})$ at six weeks of life as well as low infants' weight $(<2500)$ at birth were strongly associated with intra-partum transmission (OR $=25.5,95 \%$ CI, 1.14 - 572, $p=0.04)$, (OR = 29.6, 95\% CI, 3.2 - 273, $p=0.004)$, respectively; even though, no lower threshold value for maternal viral load below which transmission was not detected. Other factors such as vaginal mode of delivery $(\mathrm{OR}=3.3)$, prematurity $(<37$ weeks, $\mathrm{OR}=3.1)$, infant weight at birth $(<2500$, AOR $=5.0)$ were also positively associated with in-utero transmission even though they were not statistically significant in the current study (Table 4).

There were a total of five HIV infected infants at the end of this study. The overall MTCT of HIV-1 in this study was 6.2\% (95\% CI, 2 - 13.8) (Table 4). All infected infants had two positive DNA PCR results.

When combined, both non-breastfeeding and breastfeeding HIV positive mothers who had maternal viral load $\geq 24,665$ copies/ml at delivery had 27 times more likely to have HIV infected infants (OR, 27.0, 95\% CI, 3.5 $210, p=0.001$ ); compared to their counterparts. HIV positive mothers who had never used any ARV-drug during the current pregnancy had 10.1 times chance of in-utero and intra-partum transmission of HIV to their infants (OR $=10.1,95 \%$ CI, $2.2-47.4, p=0.029)$ as well as infants who weighed $<2500$ grams at birth had 18.8 times more likely to be infected $(\mathrm{OR}=18.8,95 \% \mathrm{CI}, 3.9-91.4, p=0.001)$ at the end of this study which were also statistically significant compared to those who weighed above 2500 gm. Controlling the effect of other risk factors, maternal viral load at delivery and at six weeks of birth was independently associated with both in-utero and intra-partum HIV transmission (AOR, 5.5, 95\% CI, 1.1 - 46, $p<0.001$ ). Maternal low CD4 count, higher time of labor and membrane ruptured were strongly correlated with vertical HIV transmission even if they were not statistically significant in this study (Table 4).

Of 57 breastfeeding group of the study participant mothers only 91.2\% (95\% CI, 80.7 - 97.1) had used antiretroviral drugs during the current pregnancy; 8.8\% of them did not have been treated with any ARV-drugs. All non-breastfeeding mothers had used antiretroviral drugs either they were on ART 13 (54.2\%) or they used AZT 11 (45.8\%) as prophylaxis to prevent MTCT of HIV. From 52 HIV positive breastfeeding mothers 28 (53.4\%) were on ART for at least four months and at most for two years before their current pregnancy. Whereas, 24 (46.2\%) of them had used AZT as prophylaxis. All newborn neonates had been given zidovudine oral suspension before 72 hours for seven days as prophylaxis. There was 3.8\% (95\% CI, 0.8 - 10.7), MTCT of HIV at 6 weeks of life among these infants who had used AZT oral syrups as prophylaxis.

This study indicated that giving zidovudine oral suspension to infants born to HIV positive mothers within 72 
Table 4. Major maternal and infant risk factors associated with vertical transmission of HIV-1 among infants born to HIV positive mothers at Asella and Adama hospitals, Ethiopia, 2012.

\begin{tabular}{|c|c|c|c|c|c|}
\hline Risk factors & No (\%) positive & OR (95\% CI) & $p$-value & AOR (95\% CI) & $p$-value \\
\hline \multicolumn{6}{|l|}{ Maternal } \\
\hline \multicolumn{6}{|l|}{ Viral load } \\
\hline$<$ median & & 1.0 & & & \\
\hline$\geq$ median & $2 / 74(2.7)$ & $27(3.5-210.4)$ & 0.002 & $5.5(1.1-46.3)$ & \\
\hline$\geq 4.4 \log _{10}$ & $3 / 7(42.9)$ & $12(1.54-93.5)$ & 0.018 & 4.8 & $<0.001$ \\
\hline \multicolumn{6}{|l|}{ CD4 count } \\
\hline$\geq 383 / \mu \mathrm{l}$ & $2 / 43(4.7)$ & 1.0 & & & \\
\hline$<383 / \mu l$ & 3/38 (7.9) & $1.7(2.9-9.6)$ & 0.661 & $0.8(1.4-40.0)$ & 0.891 \\
\hline \multicolumn{6}{|l|}{ Mode of delivery } \\
\hline Cesarean section & $1 / 11(9.1)$ & 1.0 & & & \\
\hline Vaginal & $4 / 70(5.7)$ & $3.3(3.0-39.8)$ & 0.348 & 6.9 & \\
\hline \multicolumn{6}{|l|}{ Duration of labor } \\
\hline$<13$ hours & 1/35 (1.9) & 1.0 & & & \\
\hline$\geq 13$ hours & $4 / 46(8.7)$ & $1.6(1.4-18.6)$ & 0.3 & & \\
\hline \multicolumn{6}{|c|}{ Duration of membrane rapture } \\
\hline$\leq 4$ hours & 1/53 (1.9) & 1.0 & & & \\
\hline$>4$ hours & 4/28 (14.3) & $4.3(0.37-50.1)$ & 0.240 & & \\
\hline \multicolumn{6}{|c|}{ Maternal prophylaxis with ARV/treatment with HAART } \\
\hline Yes & 3/76 (3.9) & 1.0 & & & \\
\hline No & $2 / 5(40)$ & $10.1(2.2-47.4)$ & 0.029 & & \\
\hline \multicolumn{6}{|l|}{ Infant } \\
\hline \multicolumn{6}{|l|}{ Prematurity (<37 weeks) } \\
\hline - No & 2/53 (3.8) & 1.0 & & & \\
\hline - Yes & $3 / 28(10.7)$ & $0.32(0.05-2.08)$ & 0.3 & & \\
\hline \multicolumn{6}{|l|}{ Birth weight } \\
\hline Normal ( $\geq 2500 \mathrm{gm})$ & $2 / 75(2.7)$ & 1.0 & & & \\
\hline Low $(<2500$ gm $)$ & $3 / 6(50)$ & $18.8(3.9-91.4)$ & 0.001 & & \\
\hline
\end{tabular}

hours of delivery reduced intra-partum/post-partum MTCT of HIV by 96.1\% (95\% CI, 89.3 - 99.2). Comparing non-ARV drug users with ARV drug user mothers during their current gestation time, MTCT of HIV was 40\% among infants born to HIV positive mothers who did not have been treated with ARV drugs (95\% CI, 5.3 - 85.3). Whereas, there was only $3.9 \%$ and $4.2 \%$ MTCT of HIV had observed among infants who born to ARV drugs user breastfeeding and non-breastfeeding mothers; respectively, at the end of this study. We could suggest that the overall MTCT of HIV would be diminished as low as 3.9\% in among breastfeeding and $4.2 \%$ among nonbreast feeding mothers if both HIV positive mothers and their new born infants could have access of using antiretroviral drugs during pregnancy and after delivery before 72 hours for mothers and their infants respectively. This number (4.2\%) seems larger among non-breastfeeding mothers due to less number of the study participants in this group.

High maternal viral load was significantly associated with both in-utero and intra-partum transmission. For in-utero transmission, adjusted for other factors, the population attributable fraction of maternal viral load above the sample median $(\geq 24,665$ copies $/ \mathrm{ml})$ was $44 \%$. Whereas, the population attributable fraction of maternal viral load above the sample median ( $\geq 9331 \mathrm{copies} / \mathrm{ml}$ ) for intra-partum/postpartum was $94 \%$ among breastfeeding mothers. Even if, the absolute CD4 cell counts did not statistically significant for in-utero and intra-partum transmission; maternal CD4 percentage $<22 \%$ at delivery and $<24 \%$ at six weeks of birth had attributed for $13 \%$ in-utero and 54\% intra-partum/postpartum MTCT of HIV in the general population; respectively, when adjusted for maternal viral load and other factors. 
In this study, there were 38 (46.9\%) and 43 (53.1\%) singleton female and male neonates with overall MTCT of HIV 10.5\% (95\% CI, 2.9 - 24.8) and 2.3\% (95\% CI, 0.1 - 12.3) born to the study participant mothers, respectively. There was $2.6 \%$ in-utero (95\% CI, 0.1 - 13.8) and 8.1\% intra-partum (95\% CI, 1.7 - 21.9) MTCT of HIV among females new born in the current study. The overall in-utero vertical transmission among male infants was 2.3\%. There was no intra-partum transmission among males. Female infants were significantly more likely to be HIV infected compared with male infants (4.94\% vs 1.23\%, respectively) (Figure 3).

\section{Discussion}

This study involved cohorts of breastfeeding and non-breastfeeding HIV positive mothers. The finding of this study could deliver crucial information regarding our PMTCT effectiveness and improvement of strategies on reducing vertical transmission of HIV in resource limited countries like Ethiopia. For the 81 mother-infant pairs with known HIV-1 DNA PCR test result at birth, at 6 weeks and at tenth weeks of life, the overall MTCT of HIV observed was 6.2\% (95\% CI, 2.1 - 3.8). Using the current definition of time of MTCT of HIV in these cohorts of HIV positive mother-infant pairs, this study confirmed that there was $2.5 \%$ (95\% CI, 0.3 - 8.6) and 3.8\% (0.8 10.7) in-utero and intra-partum transmission of HIV; respectively, in the current study. This is lower than other African studies such as Zimbabwean study [11]. However, it is comparable with study conducted in developed and some middle income countries like study conducted in France [6] [12]. It is similar with other international study [13]. Female infants were significantly more likely to be HIV infected compared with male infants (4.94\% vs $1.23 \%$, respectively). This finding is similar with a study done in Uganda [14].

In this study $40 \%$ of MTCT of HIV took place in-utero and $60 \%$ occurred in intra-partum. This is in agreement with other study conducted in France in which 37.5\% of MTCT of HIV occurred in in-utero transmission and $62.5 \%$ had taken place in intra-partum [6]. The intra-partum rate of transmission is also comparable with the study conducted in Zimbabwe that reported 69\% intra-partum rate of transmission [11]. In present study, it was found that maternal viral load at delivery was found to be independently associated both with in-utero and intrapartum residual transmission of HIV. Similar to other studies in India [6] [11]-[13], transmitting mothers had significantly higher viral load compared to non-transmitting mothers. Even though, there was no threshold level of maternal viral load could be set in this study below which no MTCT of HIV could not occur as the fact that there was transmitting mother who had viral load level as low as 95 copies $/ \mathrm{ml}$ and non-transmitting mothers who had viral load level as high as 810,237 copies/ml among the current study participant mothers. This hypothesis is consistent with some other studies that had done before in other countries like Zimbabwean study [11]; but it is contrary to study conducted in Cameroon [15]. This difference may be observed due to difference be-

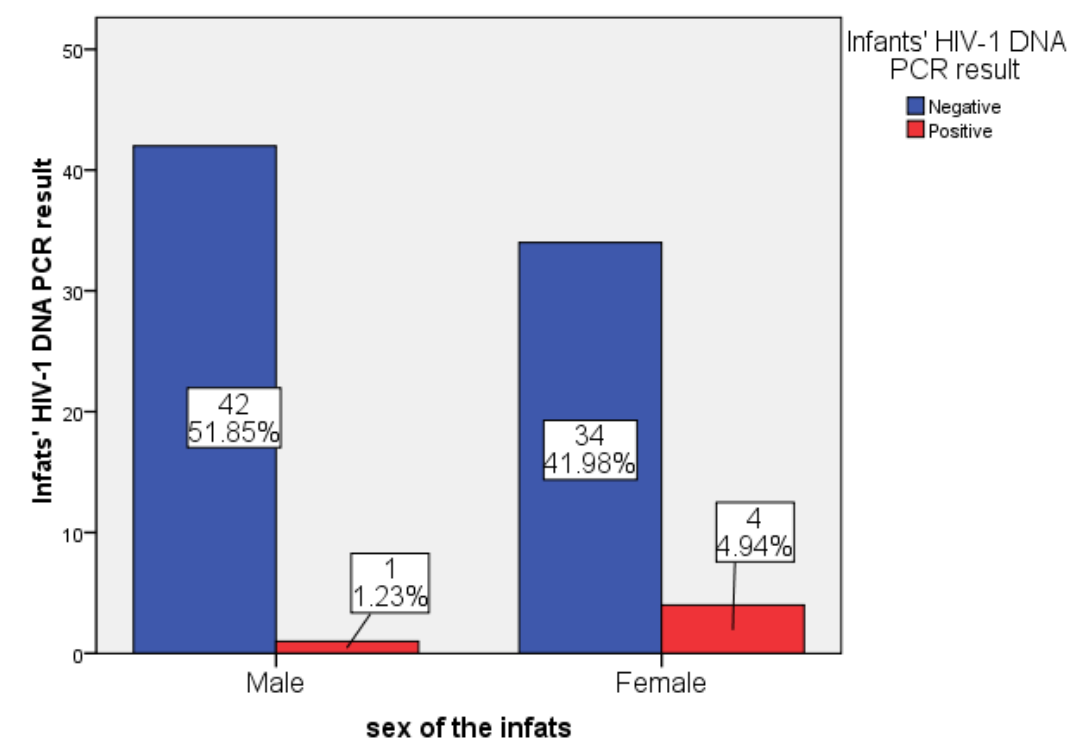

Figure 3. Distribution of MTCT of HIV-1 according to sex of the infants born to study study participant mothers at Asella and Adama hospitals, Ethiopia, 2012. 
tween the two populations in many aspects such as $93.8 \%$ of the study participants in the current study had used ARV drugs that probably result in the significant reduction of maternal viral load level. The cause of residual MTCT of HIV is remained open to debate and will be the assignment of future work in this regard to discover the mystery of HIV in residual vertical transmission. We found that maternal CD4 percentage at delivery $<22 \%$ and $<24 \%$ at six weeks of birth had attributed for $13 \%$ and $54 \%$ of in-utero and intra-partum transmission; respectively; even if, absolute maternal CD4 count was not independently and significantly associated with vertical transmission in the current study; so that, we couldn't justify further the population attributable fraction of CD4 count due to small sample size. This might be better explained in the future study.

In our study, low birth weight was associated only with intra-partum transmission. This result is also in agreement with other studies [13] [15] but it is in contrary to study conducted in Thailand that showed the association of low birth weight with in-utero transmission [16]. The main reason could be the difference between the two populations in life style, genetic variation and geographical difference. Even though, they had positive association with MTCT of HIV, other factors such as prematurity, duration of labor, duration of membrane rapture, maternal CD4 count, and mode of delivery were not independent risk factors for vertical mother-to-child transmission of HIV in the current study. This is similar with other studies [12] [13] [16] that the combined effect of these factors could increase the risk of vertical transmission; however, they might not be associated with timing of MTCT of HIV. We estimated that MTCT of HIV was $40 \%$ among breastfeeding non-ARV users, $3.9 \%$ among breastfeeding ART/prophylaxis with AZT users and 4.2\% among non-breastfeeding ARV user mothers at the end of this study. This is consistent with other studies conducted in other countries in the world [17]. Studies reported that MTCT of HIV range from 13\% - 40\% in untreated HIV positive mothers and this could be increased if they used prolonged breastfeeding mode of infant feeding. The significant reduction in vertical transmission among ARV users either in breastfeeding or non-breastfeeding group of our study participant was also consistent with other studies [18]-[20] that stated the rate of vertical transmission could be reduced significantly as low as $5.3 \%$ if single dose mother/infant NVP added to zidovudine.

In the current study, $93.8 \%$ study participant mothers, $53.9 \%$ on ART and $46.1 \%$ on prophylaxis had treated with ARV drugs according to national HIV positive mothers' ARV drugs usage/management guideline. In addition, all new born infants had been given AZT oral suspension as prophylaxis within 72 hours post delivery twice a day for 7 days. Thus, the combined mothers/infants ARV drugs treatment and shifting from short term single dose nevirapine to more potent zidovudine ARV drug could reduce MTCT of HIV from 23.8\% (95\% CI, 12.1 - 39.5) that was among the current study participant mothers during the previous pregnancy that occurred during the sdNVP era to $3.9 \%$ (95\% CI, 0.8 - 11.1) in the present study in the same population. This result could show that MTCT of HIV was about 6 times higher during the sdNVP era compared to 3.9\% vertical transmission occurred during the current new ARV drug regimens used to PMTCT of HIV. It is almost similar with other study [13] that found 3.74\% MTCT of HIV during new regimens of HAART. We could develop a simple conclusion that the current ARV drugs regimen are more efficient and effective in preventing MTCT of HIV when combined than using sdNVP to reduce vertical transmission as low as the current result. Similar conclusion was made by other investigators elsewhere throughout the world [11] [13] [21]. In the current study it was expected that MTCT of HIV would be lower among ART users than those who used prophylaxis; however, the observed outcome did not support this expectation; 53.9\% of ARV drug users of the study participant mothers were on ART for as low as four months and as high as two years before their current pregnancy which was directly related to inhibitor level of ARV drugs in the maternal circulation before the occurrence of the current pregnancy that could pass to fetus through placenta and result in significant reduction in in-utero transmission. The observed reality was in opposite when we compared ART users with those who used prophylaxis; so that, there was $4.9 \%$ (95\% CI, 0.6 - 16.5) MTCT of HIV among ART users. The rate of vertical transmission was only $2.9 \%$ (95\% CI, 0.1 - 14.9) among women who used prophylaxis. This is consistent with other study conducted in France [6], maternal viral load which might have directly associated with MTCT of HIV is independent of time of ART initiation rather other factors such as HIV strains whether susceptible or resistant to ARV drugs which we have been using and the potency of the ARV drugs in using matters the transmission.

In this study the distribution of vertical transmission between male and female infants was not balanced. In doing so, female infants were more affected than their male counterparts. The transmission rate along the sex groups was 10.5\% (95\% CI, 2.9 - 24.8) among females and 2.3\% (95\% CI, 0.1 - 12.3) among male infants. Female infants were 4.6 times more likely to be infected than males in the proportion of $2.6 \%$ in-utero and $8.1 \%$ in intra-partum transmission. This result is consistent with some study conducted in Zimbabwe [22], but in con- 
trary to other study conducted in the same country [11]. Like in other study, we could not have more justification for the difference and it would open to debate on and future works might resolve the debate in this regard. Concerning STIs and malaria infection, $19.8 \%$ and $9.9 \%$ of the study participant mothers in the current study had been treated one to two times and more than two times for STIs and Malaria, respectively. Our finding could not indicate any association of vertical HIV transmission with neither of the two infections in contrary to research finding in Cameroon [15]. This difference might be observed due to either the difference between the two population gestation age during which the infections occurred or the degree of complication relating to the infections before treatment initiated that might deteriorate the placental membrane barer which facilitates vertical HIV transmission.

\section{Conclusion}

This study indicates that maternal viral load, as measured at delivery both in breastfeeding and non-breastfeeding mothers, is independently and strongly associated with MTCT of HIV in in-utero among breastfeeding group and in intra-partum groups. So that, intervention strategies planed to reduce maternal viral load level may be successful in reducing significant number of rate of vertical transmission that can occur during in-utero, intrapartum and postpartum. Maternal viral load at six weeks of birth was also strongly associated with intra-partum/ postpartum among exclusive breastfeeding mothers that can increase the risk of HIV-1 vertical transmission among this group. The finding that as high as $40 \%$ of vertical HIV- 1 transmission occurred in-utero and $60 \%$ in intra-partum provided that effective preventive services given at the end of pregnancy can result in significant reduction of transmission both during in-utero and intra-partum as well as overall transmission risk. The result of this study also supports the notion that combined therapy is more effective than single dose to reduce vertical HIV-1 transmission. Based on the finding of this study, we recommend that combined ARV-therapy is more effective than single dose for valuable reduction of vertical HIV-1 transmission if the benefit outweighs the risk both for mothers and their fetus/new born infants.

\section{References}

[1] De Cock, K.M., Fowler, M.G., Mercier, E., et al. (2000) Prevention of Mother-to-Child HIV Transmission in Resource-Poor Countries: Translating Research into Policy and Practice. JAMA, 283, 1175-1182. http://dx.doi.org/10.1001/jama.283.9.1175

[2] Mullick, S., Watson-Jones, D., Beksinska, M. and Mabey, D. (2005) Sexually Transmitted Infections in Pregnancy: Prevalence, Impact on Pregnancy Outcomes, and Approach to Treatment in Developing Countries. Sexually Transmitted Infections, 81, 294-302. http://dx.doi.org/10.1136/sti.2002.004077

[3] UNAIDS (2010) Report on the Global AIDS Epidemic. UNAIDS, Geneva. http://data.unaids.org/pub/Report/2009/2009 epidemic update en.pdf

[4] WHO (2010) Antiretroviral Drugs for Treating Pregnant Women and Preventing HIV Infection in Infants. Recommendations for a Public Health Approach (2010 Version). WHO, Geneva. http://www.who.int/hiv/pub/mtct/antiretroviral2010/en/index.html

[5] Tubiana, R., Chenadec, J.L., Rouzioux, C., Mandelbrot, L., Hamrene, K., Dollfus, C., et al. (2010) Factors Associated with Mother-to-Child Transmission of HIV-1 Despite a Maternal Viral Load 500 Copies/mL at Delivery: A CaseControl Study Nested in the French Perinatal Cohort (EPF-ANRS CO1). Clinical Infectious Diseases, 50, 585-596. http://dx.doi.org/10.1086/650005

[6] Mofenson, L.M. (2002) US Public Health Service Task Force Recommendations for Use of Antiretroviral Drugs in Pregnant HIV-1-Infected Women for Maternal Health and Interventions to Reduce Perinatal HIV-1 Transmission in the United States. MMWR-Recommendations and Reports, 51, 1-38.

[7] Chinnock, P., Siegfried, N. and Clarke, M. (2005) Is Evidence-Based Medicine Relevant to the Developing World. PLOS Medicine, 2, Article ID: e277.

[8] Okonji, J.A., Zeh, C., Weidle, P.J., Williamsin, J., Akott, B., Masaba, R., et al. (2012) CD4, Viral Load Response and Adherence among Antiretroviral-Naive Breastfeeding Women Receiving Triple Antiretroviral Prophylaxis for Prevention of Mother-to-Child Transmission of HIV in Kisumu, Kenya. Journal of Acquired Immune Deficiency Syndromes, 61, 249-257. http://dx.doi.org/10.1097/QAI.0b013e318262514f

[9] Charurat, M., Datong, P., Matawal, B., Ajene, A., Blattner, W. and Abimiku, A. (2009) Timing and Determinants of Mother-to-Child Transmission of HIV in Nigeria. International Journal of Gynecology Obstetrics, 106, 8-13. http://dx.doi.org/10.1016/j.ijgo.2009.02.017 
[10] Jamieson, D.J., Sibailly, T.S., Sadek, R., Roels, T.H., Ekpini, E.R., Boni-Ouattara, E., et al. (2003) HIV-1 Viral Load and Other Risk Factors for Mother-to-Child Transmission of HIV-1 in a Breast-Feeding Population in Cote d'Ivoire. Journal of Acquired Immune Deficiency Syndromes, 34, 430-436. http://dx.doi.org/10.1097/00126334-200312010-00011

[11] Duri, K., Gumbo, F.Z., Kristiansen, K.I., Kurewa, N.E., Mapingure, M.P., Rusakaniko, S., et al. (2010) Antenatal HIV-1 RNA Load and Timing of Mother to Child Transmission; A Nested Case-Control Study in a Resource Poor Setting. Virology Journal, 7, 176. http://dx.doi.org/10.1186/1743-422X-7-176

[12] Gupta, A., Gupte, N., Sastry, J., Bharucha, K.E., Bhosale, R., Kulkarni, P., et al. (2007) Mother-to-Child Transmission of HIV among Women Who Chose Not to Exclusively Breastfeed Their Infants in Pune, India. Indian Journal of Medical Research, 126, 131-134

[13] Delicio, A.M., Milanez, H., Amaral, E., Morais, S.S., Lajos, G.J., Silva, J., et al. (2011) Mother-to-Child Transmission of Human Immunodeficiency Virus in a Ten Years Period. Reproductive Health, 8, 35. http://www.reproductive-health-journal.com/content/8/1/35 http://dx.doi.org/10.1186/1742-4755-8-35

[14] Brahmbhatta, H., Kigozib, G., Serwaddac, D., Wabwire-Mangend, F., Sewankamboe, N., Wawera, M., et al. (2009) Is the Risk of Mother-to-Child Transmission of HIV Higher among Female Compared with Male Infants? A Case of Rakai, Uganda. The Pediatric Infectious Disease Journal, 4, 275-279.

[15] Ayouba, A., Nerrienet, E., Menu, E., Lobé, M.M., Thonnon, J., Leke, R.J., et al. (2003) Mother-to-Child Transmission of Human Immunodeficiency Virus Type 1 in Relation to the Season in Yaoundé, Cameroon. The American Journal of Tropical Medicine and Hygiene, 69, 447-449.

[16] Mock, P.A., Shaffer, N., Bhadrakom, P., Siriwasin, W., Chotpitayasunondhi, T., Chearskul, S., et al. (1999) Maternal Viral Load and Timing of Mother-to-Child HIV Transmission, Bangkok, Thailand. Bangkok Collaborative Perinatal HIV Transmission Study Group. AIDS, 13, 407-414. http://dx.doi.org/10.1097/00002030-199902250-00014

[17] Townsend, C.L., Cortina-Borja, M., Peckham, C.S., de, R.A., Lyall, H. and Tookey, P.A. (2008) Low Rates of Mother-to-Child Transmission of HIV Following Effective Pregnancy Interventions in the United Kingdom and Ireland, 2000-2006. AIDS, 22, 973-981. http://dx.doi.org/10.1097/QAD.0b013e3282f9b67a

[18] Cooper, E.R., Charurat, M., Mofenson, L., Piatt, J., Diaz, C., Hayani, K., et al. (2002) Women and Infants’ Transmission Study Group: Combination Antiretroviral Strategies for the Treatment of Pregnant HIV-1-Infected Women and Prevention of Perinatal HIV-1 Transmission. Journal of Acquired Immune Deficiency Syndromes, 29, 484-494. http://dx.doi.org/10.1097/00126334-200204150-00009

[19] Fowler, M.G., Mofenson, L., McConnell, M., et al. (2003) The Interface of Perinatal HIV Prevention, Antiretroviral Drug Resistance, and Antiretroviral Treatment: What Do We Really Know? Journal of Acquired Immune Deficiency Syndromes, 34, 308-311. http://dx.doi.org/10.1097/00126334-200311010-00009

[20] Abrams, E.J. (2004) Prevention of Mother to Child Transmission of HIV Successes, Controversies and Critical Questions. Aids Reviews, 6, 131-143.

[21] Bedri, A., Gudetta, B., Isehak, A., Kumbi, S., Lulseged, S., Mengistu, Y., et al. (2008) Extended-Dose Nevirapine to 6 Weeks of Age for Infants to Prevent HIV Transmission via Breastfeeding in Ethiopia, India, and Uganda: An Analysis of Three Randomized Controlled Trials. Lancet, 372, 300-313. http://dx.doi.org/10.1016/S0140-6736(08)61114-9

[22] Piwoz, E.G., Humphrey, J.H., Tavengwa, N.V., Iliff, P.J., Marinda, E.T., Zunguza, C.D., et al. (2007) The Impact of Safer Breastfeeding Practices on Postnatal HIV-1 Transmission in Zimbabwe. American Journal of Public Health, 97, 1249-1254. http://dx.doi.org/10.2105/AJPH.2006.085704 